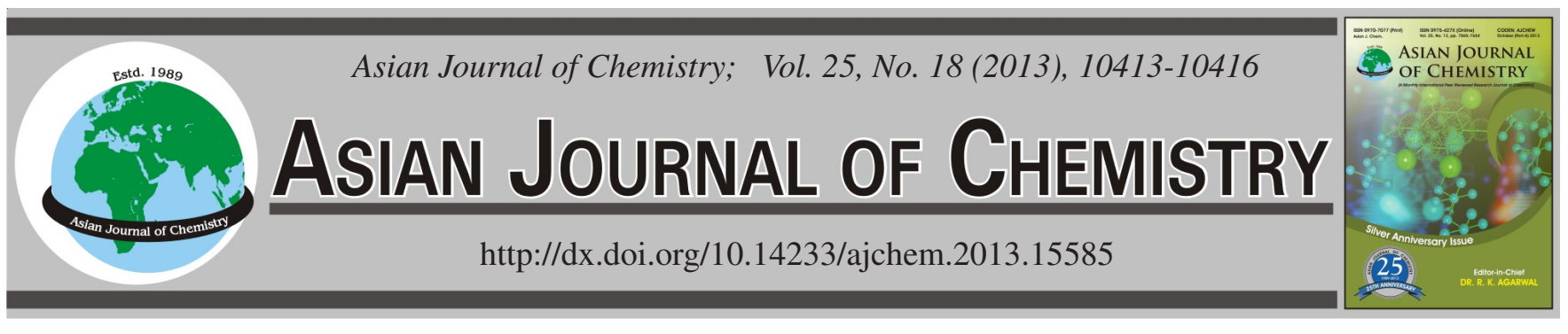

\title{
Fast Analysis of Puerarin and Daidzein in Radix puerariae and It's Medicinal Preparation by Micellar Electrokinetic Capillary Chromatography
}

\author{
K. JIA ${ }^{1}$, J. WANG ${ }^{2}$ and K. $\mathrm{AN}^{3, *}$
}

\author{
${ }^{1}$ Yucheng People's Hosptical, Kaituo Road 753, Yucheng 251200, P.R. China \\ ${ }^{2}$ Dezhou People's Hosptical, Sanba Road 1, Dezhou 253045, P.R. China \\ ${ }^{3}$ Shandong Academy of Medical Sciences, Jingshi Road 18877, Jinan 250062, P.R. China \\ *Corresponding author: Tel. 86531 82919831; E-mail: ka20130327@163.com
}

\begin{abstract}
A method was developed to determine puerarin and daidzein in Radix puerariae and it's medicinal preparation Gegen qinlianpian by micellar eletrokinetic capillary chromatography. The analytical conditions were optimized by investigating the effects of buffer $\mathrm{pH}$ and concentration, sodium dodecyl sulfate concentration and $\beta$-cyclodextrin. Under the optimized conditions, the two analytes were well separated in $5 \mathrm{~min}$. A good linear relationship between the peak height and concentration was found in the $0.02-1.20$ and $0.02-1.00 \mathrm{mg}$ $\mathrm{mL}^{-1}$ concentration range for puerarin and daidzein, respectively. The relative standard deviation based on migration time and peak height were 0.51 and $1.82 \%$ for puerarin, 0.45 and $1.68 \%$ for daidzein, respectively. The detection limits were 0.005 and $0.004 \mathrm{mg} \mathrm{mL}^{-1}$ for puerarin and daidzein, respectively.
\end{abstract}

Key Words: Micellar electrokinetic capillary chromatography, Radix puerariae, Gegen qinlianpian, Puerarin, Daidzein.

ட - - - - - - - - - - - - - - - - - - - - - - - -

\section{INTRODUCTION}

Due to their great physiological and pharmacological effects, the analysis of flavonoids in traditional Chinese medicine has drawn much attention ${ }^{1,2}$. Radix puerariae, refered to as "Ge-gen" in Chinese, is an important crude herb used in Chinese medicine ${ }^{3}$. It has the functions of relieving the muscles, removing fever, promoting the production of body fluid, exposing the exthanthema and relieving diarrhea and can be used to treat common cold, influenza, wrist or shoulder stiffness and other symptoms ${ }^{3}$. It has been reported that Radix puerariae contains puerarin, daidzein (Fig. 1) and other flavonoids ${ }^{3}$. At present, the common method for the components analysis of Radix puerariae and its medicinal preparations are thin layer chromatography (TLC) ( $^{4,5}$ and high perforemance liquid chromatography (HPLC) ${ }^{6-8}$. However, neither of the above two methods has enough resolving power or separation efficiency.

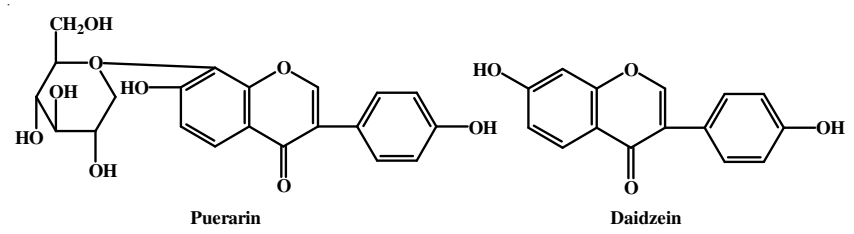

Fig. 1. Structures of the analytes
Compared to the above two methods, high performance capillary electrophoresis (HPCE) has the advantages of fast analysis speed, high efficiency, low consumption of samples and the reagents and has been more and more widely used in the components analysis of Chinese traditional medicine ${ }^{9-18}$. Micellar electrokinetic capillary chromatography (MEKC) is a capillary electrophoretic separation mode based on the distribution of analytes between the micellar pseudo stationary phase and the aqueous phase. It is the combination of capillary zone electrophoresis and liquid chromatography and can not only separate the electroneutral compounds but can also improve the separation of ionic compound. In this paper, after investigation of the effects of buffer $\mathrm{pH}$ and concentration, applied voltage, sodium dodecyl sulfate concentrationand and $\beta$-cyclodextrin, a micellar electrokinetic capillary chromatographic method was established for the fast determination of puerarin and daidzein in Radix puerariae and its medicinal preparation.

\section{EXPERIMENTAL}

Capillary electrophoresis experiments were performed on a 1229 type HPCE Analytical System (Beijing Institute of New Technology and Application, Beijing, China) with a fixed wavelength UV detector. A fused silica capillary (Yongnian Optical Fiber Factory, Hebei, China) of $42 \mathrm{~cm} \times 50 \mathrm{~mm}$ was 
used as a separation tube. On-column detection was performed at the cathode on a ultra-violet detector at $254 \mathrm{~nm}$. Electropherograms were recorded on a analytical star integrator. The separation voltage was $16 \mathrm{kV}$. The capillary electrophoresis operation was performed at ambient temperature. Samples were injected with the hydrostatic mode at anode for $8 \mathrm{~s}(10 \mathrm{~cm})$. A pHS-3C pH meter (Leici Instrumentation Factory, Shanghai, China) was used, A H 6605 ultrasonic cleanser (Wuxi Electronic Equipment Co., Wuxi, China) was used for ultrasonication.

Puerin and daidzein were purchased from Sigma (St. Louis, MO, USA), all the above reagents were used as received. $\beta$-Cyclodextrin was purchased from the Development Center of Special Chemical Reagents in North China. Sodium dodecyl sulfate (SDS) was from Beijing Chemical Plant. All aqueous solutions were prepared in doubly distilled water. Other chemicals were of analytical reagent grade. Radix puerariae was purchased from Tongrentang Drugstore in Beijing, China. Gegen Qinlianpian was from Jiashitang Drugstore in Beijing, China.

Procedure: Stock solutions of puerarin and daidzein were prepared in ethanol. All the solutions were kept in a $4{ }^{\circ} \mathrm{C}$ refrigerator and were stable for at least 1 month. More dilute solutions were freshly prepared from the stock solutions with ethanol when needed. All the solutions was filtered through a $0.45 \mu \mathrm{m}$ membrane filter and degassed by ultrosonication for approximately $10 \mathrm{~min}$ before use. The running buffer consisted of $20 \mathrm{mmol} \mathrm{L}^{-1}$ Borax- $\mathrm{KH}_{2} \mathrm{PO}_{4}$ (pH 9.0) and $30 \mathrm{mmol}$ $\mathrm{L}^{-1}$ sodium dodecyl sulfate. The capillary was conditioned daily by washing with $0.1 \mathrm{~mol} \mathrm{~L}^{-1} \mathrm{NaOH}$, doubly distilled water (10 $\mathrm{min})$ and the running buffer (10 min) sequentially. Between consecutive analysis, the capillary was flushed with water (1 min), $0.1 \mathrm{~mol} \mathrm{~L}^{-1}$ sodium hydroxide (1 min), then with water ( $2 \mathrm{~min}$ ) and finally with the running buffer ( $3 \mathrm{~min}$ ) in order to improve the migration time and peak height repeatability. Peak identification of the analytes peaks in the electropherogram of real sample was achieved by standard addition method as well as by comparing migration time between that in the real sample and in the standard solution.

Sample preparation: Radix puerariae was dried at $60{ }^{\circ} \mathrm{C}$ for $4 \mathrm{~h}$ in an oven and then pulverized, an accurate weight amount $(1.0804 \mathrm{~g})$ of the powder was refluxed with $50 \mathrm{~mL}$ of $80 \%(\mathrm{v} / \mathrm{v})$ ethanol for $5 \mathrm{~h}$ in a $75^{\circ} \mathrm{C}$ water bath. After cooling, the mixture was filtered through a paper filter and the residue was washed three times with $10 \mathrm{~mL}$ of $80 \%(\mathrm{v} / \mathrm{v})$ ethanol. The extract and washings were combined and concentrated to about $10 \mathrm{~mL}$ under vacuum. This solution was diluted to $25 \mathrm{~mL}$ in a brown volumetric flask with $80 \%(\mathrm{v} / \mathrm{v})$ ethanol.

Gegen qinliangpian tablet were pulverized, an accurate weight amount $(0.9991 \mathrm{~g})$ of the powder was put into $20 \mathrm{~mL}$ of $80 \%(\mathrm{v} / \mathrm{v})$ aqueous ethanol and soaked for $4 \mathrm{~h}$, then ultrasonicated for $2 \mathrm{~h}$. The mixture was filtered through a paper filter and the residue was washed twice with $2 \mathrm{~mL}$ of $80 \%$ aqueous ethanol. The extract and washings were combined and diluted to $25 \mathrm{~mL}$ in a brown columetric flask with $80 \%$ $(\mathrm{v} / \mathrm{v})$ aqueous ethanol and was filtered through a $0.45 \mu \mathrm{m}$ membrane filter before use.

\section{RESULTS AND DISCUSSION}

Effect of buffer $\mathbf{p H}$ and concentration: Under the condition of $20 \mathrm{mmol} \mathrm{L}^{-1}$ Borax- $\mathrm{KH}_{2} \mathrm{PO}_{4}$ and $30 \mathrm{mmol} \mathrm{L}^{-1}$ sodium dodecyl sulfate, the effect of buffer $\mathrm{pH}$ on the separation was investigated in the $\mathrm{pH} 8-10$ range. As shown in Fig. 2(a), with the increase of the buffer $\mathrm{pH}$, the migration time of puerarin increased, while that of daidzein decreased. The resolution of the two analyes decreased with the increase of the buffer $\mathrm{pH}$. At the same time, the peak shape of the two analytes were very poor at low $\mathrm{pH}$, it improved with the increase of buffer $\mathrm{pH}$, this improvement became trivial after $\mathrm{pH}$ 9, so $\mathrm{pH} 9$ was selected for determination.
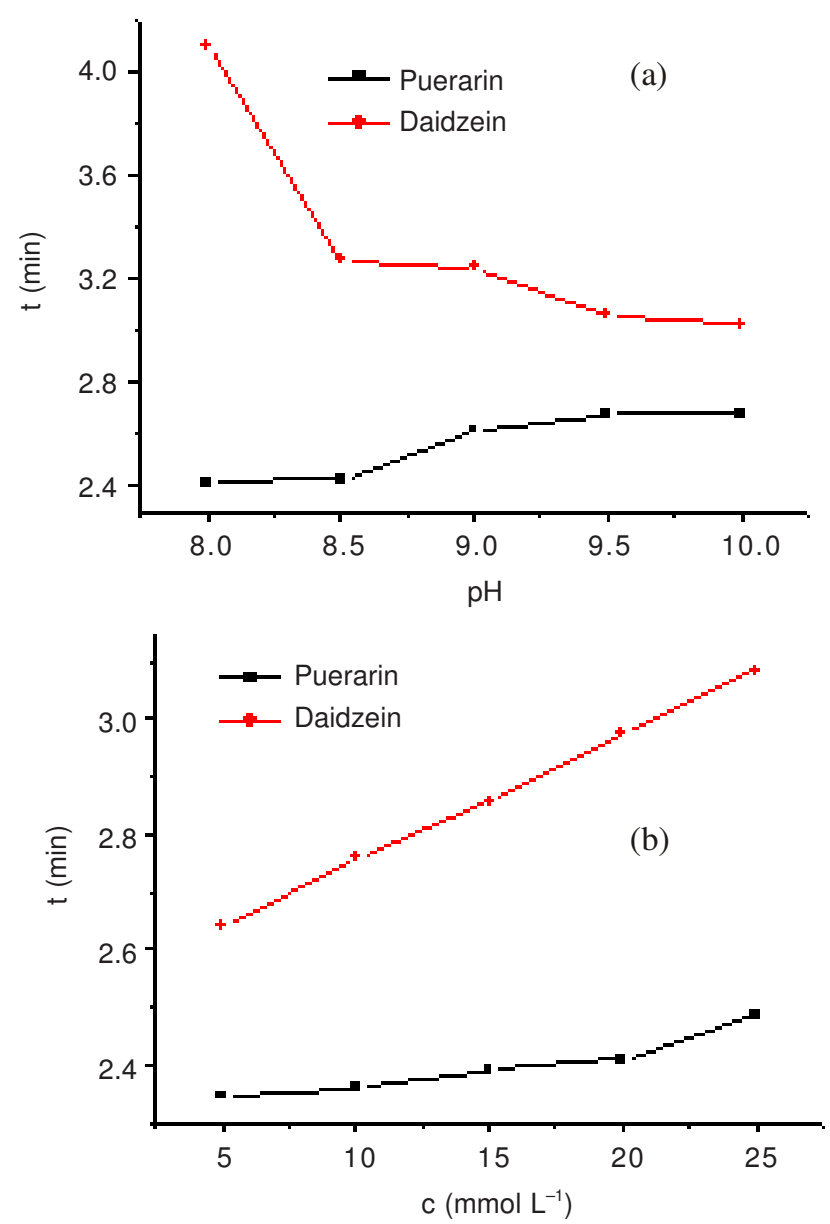

Fig. 2. Effects of buffer acidity (a) and buffer concentration (b) on the migration of the analytes

Keep the buffer $\mathrm{pH}$ at $\mathrm{pH} 9$ and other conditions the same as the $\mathrm{pH}$ optimization, the influence of buffer concentration was investigated in the $5-25 \mathrm{mmol} \mathrm{L}^{-1}$ concentration range. As indicated in Fig. 2(b), the migration time and the separation of the two analytes increases with increasing buffer concentration. A good separation was achieved between puerarin and daidein when the buffer concentration is higher than $20 \mathrm{mmol}$ $\mathrm{L}^{-1}$, in consideration of resolution and analysis time, $20 \mathrm{mmol}$ $\mathrm{L}^{-1}$ Borax $-\mathrm{KH}_{2} \mathrm{PO}_{4}$ was selected as a compromise.

Effect of applied voltage: The effect of applied voltage on the separation of the analytes was examined in the range of $12-20 \mathrm{kV}$ at $\mathrm{pH}$ 9. The results showed that the migration time of the two analytes decrease with the increase of applied voltage, which results in shorter analysis time. While at the same time, the resolution of the two analytes reduced. The peak heights increase with the increase of applied voltage in the $12-16 \mathrm{kV}$ 
range, after the applied voltage exceeded $16 \mathrm{kV}$, it decreased. Furthermore, the increase of applied voltage could lead to pronounced Joule heating, which would result in deteriorated separation efficiency and detection limit. Taking resolution, peak height, separation efficiency and detection limits into consideration, $16 \mathrm{kV}$ was selected for experiment.

Effect of SDS concentration: To investigate the effect of SDS concentration on migration time and separation efficiency, $20 \mathrm{mmol} \mathrm{L}{ }^{-1}$ Borax- $\mathrm{KH}_{2} \mathrm{PO}_{4}$ at $\mathrm{pH} 9$ with different SDS concentration from $0-40 \mathrm{mmol} \mathrm{L}^{-1}$ was used to separate the analytes. The results has been shown in Fig. 3, where the migration time was plotted against SDS concentration, without SDS, the resolution of the two analytes were poor. An increase in the migration time and resolution of the two analytes was observed when the SDS concentration increased. The reason for the increase may be that at higher SDS concentrations the phase ratio of the micelle to the aqueous phase would be larger. So the probability of the solubilization of the constitutents by the micelles would be higher, resulting in an increase in the migration time for these compounds ${ }^{19}$. A good separation for the two analytes were found when SDS concentration was higher than $30 \mathrm{mmol} \mathrm{L}^{-1}, 30 \mathrm{mmol} \mathrm{L}^{-1} \mathrm{SDS}$ was selected for the shorter analysis time.

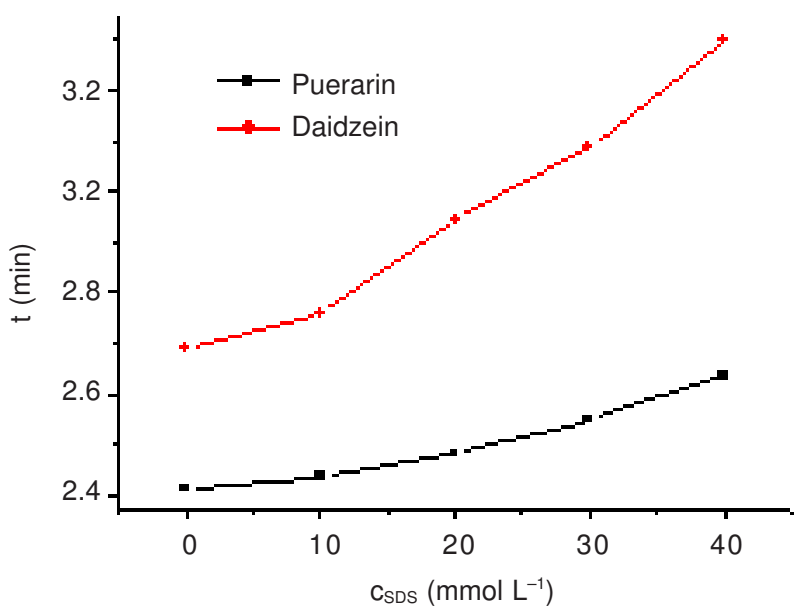

Fig. 3 Effects of SDS concentration on the migration of the analytes

Effect of $\boldsymbol{\beta}$-cyclodextrin: In micellar electrokinetic capillary chromatography analysis, incorporation of $\beta$-cyclodextrin into the running buffer can improve the separation selectivity and resolution for some analytes due to the ability of $\beta$-cyclodextrin to include selectively of these molecules in its hydrophobic cavity ${ }^{20}$. In an attempt to achieve additional improvement of the resolution of the analytes, the effect of $0-10 \mathrm{mmol}$ $\mathrm{L}^{-1} \beta$-cyclodextrin added into the BGE was tested. The results was shown in Fig. 4, with the increase of the $\beta$-cyclodextrin concentration, the migration time of the two analytes decreased. At the same time, the separation of the two analytes reduced with the increase of the concentration of $\beta$-cyclodextrin and some other constituents will co-migrate with the analytes. Therefore, $\beta$-cyclodextrin was not included in the buffer system in the further experiments.

Analytical Characterization: Under the optimized conditions, good separation of the two analytes was achieved

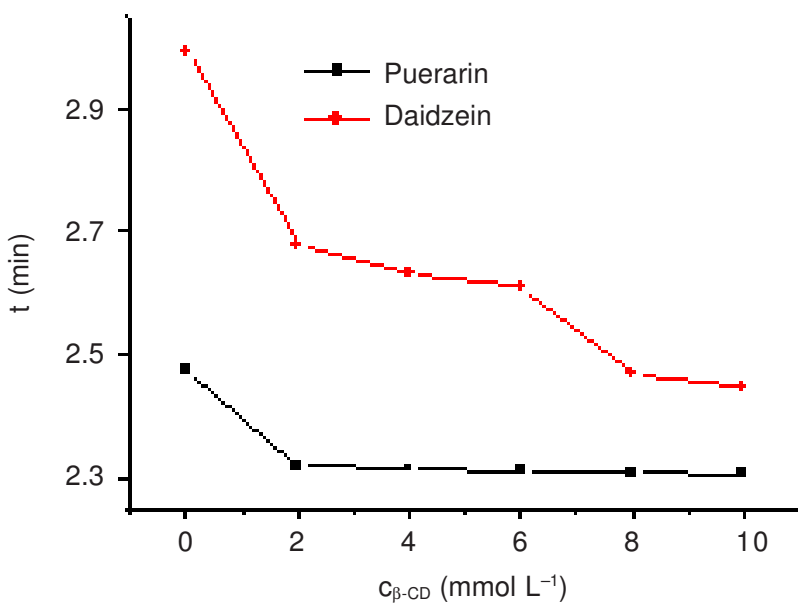

Fig. 4. Effects of $\beta$-cyclodextrin concentration on the migration of the analytes

in $5 \mathrm{~min}$, the separated electropherogram of standard mixture has been shown in Fig. 5. The linearity of the two analytes in standard solutions was investigated. The calibration graphs were plotted by concentration $\left(\mathrm{x}, \mathrm{mg} \mathrm{mL}^{-1}\right)$ against peak height $(\mathrm{y}, \mathrm{mV})$. The calibration plots were linear in the concentration range of $0.02-1.20$ and $0.02-1.00 \mathrm{mg} \mathrm{mL}^{-1}$ for puerarin and daidzein, respectively. The regression equations of these curves and their correlation coefficients were calculated as follows: puerarin, $\mathrm{y}=98.039 \mathrm{x}-0.046(\mathrm{r}=0.9996)$ and daidzein, $\mathrm{y}=$ $126.212 x+0.085(r=0.9998)$. The reproducibility evaluated by consecutive injection of a standard solution containing 0.10 $\mathrm{mg} \mathrm{mL} \mathrm{m}^{-1}$ puerarin and daidzein 5 times. The variation in migration time and peak height were $0.51 \%, 1.82 \%$ for puerarin and 0.45 and $1.68 \%$ for daidzein, respectively. The detection limits estimated based on a signal to noise ratio of 3 , were 0.005 and $0.004 \mathrm{mg} \mathrm{mL}^{-1}$ for puerarin and daidzein, respectively.

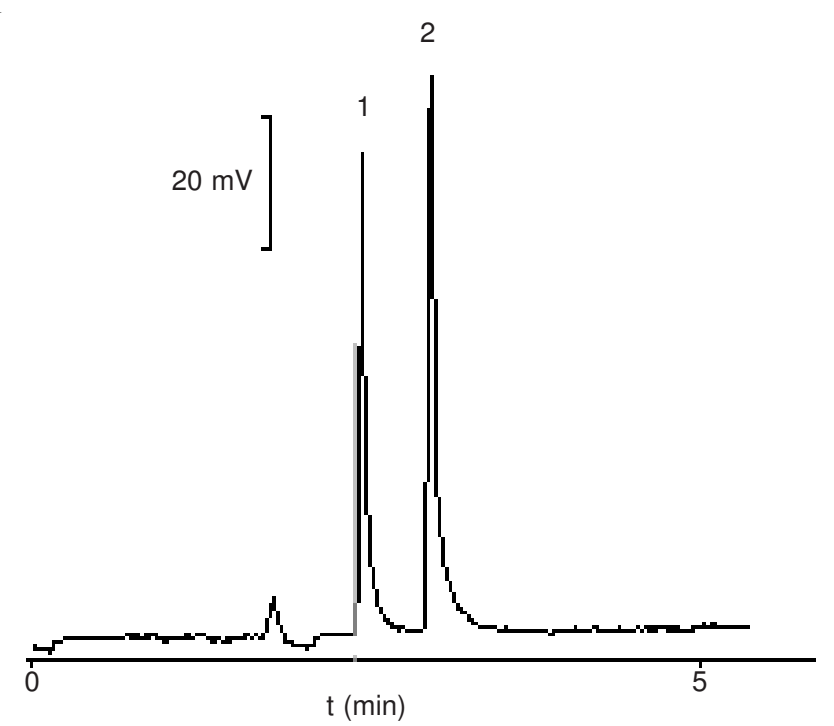

Fig. 5 Electropherograms of standard mixture: 1. puerarin, 2. daidzein. Analytical conditions: buffer: $20 \mathrm{mmol} \mathrm{L}{ }^{-1}$ Borax ( $\mathrm{pH} 9.0$ )-30 mmol $\mathrm{L}^{-1} \mathrm{SDS}$, applied voltage: $16 \mathrm{kV}$

Sample analysis: The developed method was applied to determine puerarin and daidzein in Radix puerariae and its medicinal preparation Gegen qinlianpian. Typical electro- 
pherograms of the samples were shown in Fig. 6(a) and 6(b). The assay results were shown in Table-1, which were satisfactory. Accuracy of the method was determined by adding suitable amounts of the analytes of known concentration to the extracts of samples. Recovery experiments were performed 5 times by adding $0.5 \mathrm{mg} \mathrm{mL}^{-1}$ of puerarin and daidzein into the extract of Radix puerariae and Gegen qinlianpian, the recoveries of puerarin and daidzein were 94.5-102.8 \%, 96.5-104.5\% and 96.5-103.4\%, 95.4-101.5\%, respectively.

\begin{tabular}{cccc}
\multicolumn{4}{c}{ TABLE-1 } \\
\multicolumn{4}{c}{ RESULTS OF SAMPLE ANALYSIS $(\mathrm{n}=5)$} \\
\hline Sample & Component & Content $\left(\mathrm{mg} \mathrm{g}^{-1}\right)$ & R.S.D. $(\%)$ \\
\hline \multirow{2}{*}{ Radix puerariae } & Puerarin & 18.63 & 1.61 \\
& Daidzein & 1.79 & 2.42 \\
\hline \multirow{2}{*}{ Gegen qinlianpian } & Puerarin & 7.89 & 2.45 \\
& Daidzein & 0.91 & 2.30 \\
\hline
\end{tabular}
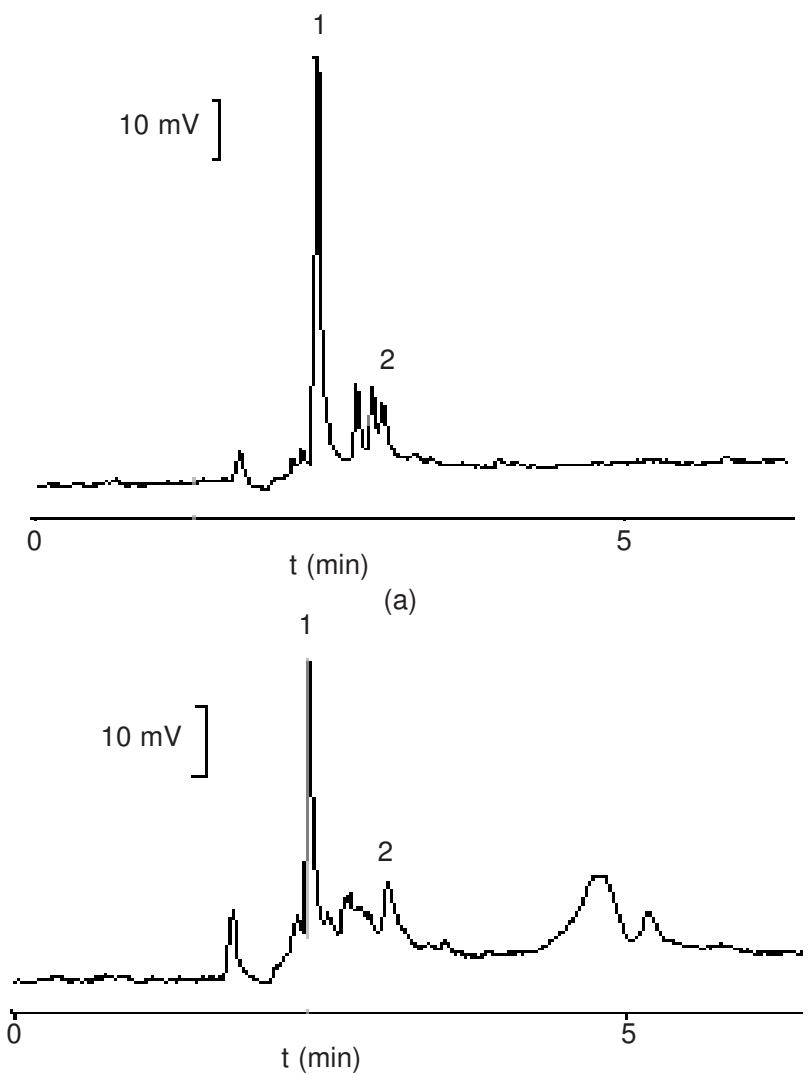

(b)

Fig. 6 Electropherograms of ethanol extract of Radix puerariae (a) and Gegen qinlianpian (b) :1. puerarin, 2. daidzein. Analytical conditions are the same to Fig. 5

\section{Conclusion}

After systematic investigation of the analytical conditions of buffer concentration and $\mathrm{pH}$, applied voltage, SDS concentration and $\beta$-cyclodextrin, a method of determination of puerarin and daidzein in Radix puerariae and its medicinal preparation Gegen qinlianpian by micellar electrokinetic capillary chromatography was developed. The assay results showed that the developed method was rapid, accurate, reproducible and enjoyed the potential to be used for quality control of this kind of medicine.

\section{ACKNOWLEDGEMENTS}

This work was supported by the the Key Technologies R\&D Programme of Shandong Province (2010GSF10279).

\section{REFERENCES}

1. H.B. Xiao, M. Krucker, K. Albert and X.M. Liang, J. Chromatogr. A, 1032, 117 (2004).

2. M. Ye, Y. Li, Y. Yan, H. Liu and X. Ji, J. Pharm. Biomed. Anal., 28, 621 (2002).

3. T. Huang, Z. Ding and S. Zhao, Modern Compendium Material Medica, Chinese Medical Science and Technology Press, Beijing, p. 1621 (2001).

4. X. Shang, C. Dong, Y. Feng, P. Wang and C. Liu, Chin. J. Anal. Chem., 29, 115 (2001).

5. Z. Zhang, Q. Liu, Q. He and Z. Gao, Chin. J. Anal. Chem., 30, 327 (2002).

6. S. Lin, Y. He, M. Nan, T. Wang and Q. Zhao, Lishizhen Med. Mater. Med. Res., 17, 205 (2006).

7. Y. Zhong, J. Wang, S. Yang, W. Tang and C. Zuo, Lishizhen Med. Mater. Med. Res., 11, 1059 (2000).

8. B. Chen, H. Zhao and Y. Yan, Chin. J. Food Sci., 22, 64 (2001).

9. Q.C. Chu, W.Q. Qu, Y.Y. Peng, Q.H. Cao and J.N. Ye, Chromatographia, 58, 67 (2003).

10. L. Suntornsuk, S. Kasemsook and S. Wongyai, Electrophoresis, 24, 1236 (2003).

11. Q. Chu, L. Fu, Y. Cao and J.Ye, Phytochem. Anal., 17, 176 (2006).

12. L. Hua, Z. Peng, L. S. Chia, N.K. Goh and S.N. Tan, J. Chromatogr. A, 909, 297 (2001).

13. M.E. Yue, T.F. Jiang and Y.P. Shi, Talanta, 62, 695 (2004).

14. Y. Lu, Y.Li and H. Li, J. Chem. Soc. Pak., 30, 829 (2008).

15. Y. Lu and H. Li, Asian J. Chem., 21, 3869 (2009).

16. Z. Ning, H. Zhong, C. Kong and Y. Lu, Asian J. Chem., 24, 2965 (2000).

17. H. Zhong, Q.Yao, M.C. Breadmore, Y. Li and Y. Lu, Analyst, 136, 4486 (2011).

18. J. Cao, H. Qu and Y. Cheng, Electrophoresis, 31, 1689 (2010).

19. Y.M. Liu and S.J. Sheu, Anal. Chim. Acta, 288, 221 (1994).

20. Y. Deng and J. He, High Perforemance Capillary Electrophoresis, Academic Press, Beijing, p. 133 (1995). 